\title{
Medium effects on spin observables of proton knockout reactions
}

\author{
G. Krein \\ Instituto de Física Teórica-Universidade Estadual Paulista, Rua Pamplona, 145-01405-000 São Paulo, Brazil \\ Th. A. J. Maris, B. B. Rodrigues, and E. A. Veit \\ Instituto de Física-Universidade Federal do Rio Grande do Sul, Caixa Postal, 15051-91501-970 Porto Alegre, Brazil
}

(Received 12 July 1994)

\begin{abstract}
Medium modifications of the properties of bound nucleons and mesons are investigated by means of intermediate energy quasifree proton knockout reactions with polarized incident protons, by comparison of quantities which are insensitive against wave distortions. The sensitivity of the spin observables of the quasifree proton-proton cross section to modifications of the nucleon and meson properties is studied using the Bonn one-boson exchange model of the nucleon-nucleon interaction. A method proposed to extract the $p p$ analyzing power in medium from the $(\vec{p}, 2 p)$ asymmetries indicates a reduction of this quantity compared to its free space value. This reduction is linked to modifications of masses and coupling constants of the nucleons and mesons in the nucleus. The implications of these modifications for another spin observable to be measured in the future are discussed.
\end{abstract}

PACS number(s): 25.40.Cm, 21.30.+y

\section{INTRODUCTION}

In recent years the question of medium modifications of nucleons and mesons properties has received a great deal of attention [1-12]. There have been speculations on modifications of nucleon and meson masses and sizes, and of meson-nucleon coupling constants. These speculations have been motivated from a variety of theoretical points of view, which include renormalization effects due to strong relativistic nuclear fields, deconfinement of quarks, and chiral symmetry restoration. Independently of the theoretical explanation, it is important to have different experiments which might provide information on this issue.

Quasifree $(x, x N)$ reactions represent probably the most direct manner to measure single-particle properties in nuclei. Hence, they are a suitable tool to observe medium modifications of nucleons and mesons properties and their consequences on physical observables of these experiments. In this paper we propose to use quasifree $(\vec{p}, 2 p)$ reactions $^{1}$ with polarized incident protons to investigate medium modifications of bound nucleons.

In quasifree $(p, 2 p)$ scattering an incident proton of medium energy (200-1000 MeV) knocks out a bound proton [13]. The only violent interaction of this process occurs between the incident particle and the ejected one. The wave functions of incoming and outcoming nucleons are just distorted while traversing the nucleus. By measuring in coincidence the energies and momenta of the emerging nucleons, these processes provide direct information on single-particle separation energy spectra and momentum distributions. In the last three decades

\footnotetext{
${ }^{1}$ The arrow over $p$ indicates a polarized incident beam.
}

quasifree scattering experiments have been performed with this basic purpose. For an overview of this topic see Refs. [14,15].

The formalism generally used to describe quasifree reactions is based on the impulse approximation to describe the violent quasifree collision, whereas initial- and final-state interactions, or distortions, are described by complex optical potentials. The cross section of $(p, 2 p)$ reactions is sensitively dependent on these distortions. In particular, the imaginary part of the optical potentials, representing the multiple scattering, may reduce the quasifree cross section by an order of magnitude. As a consequence a relatively small change in the somewhat uncertain imaginary optical potentials may spoil a good description of an experimental result. In other words, a good fit to an experimental result may partly be due to a fortunate adjustment of the distorting potential. A new perspective in this field has been opened by the possibility of exploring spin and isospin degrees of freedom $[16,17]$, especially due to the fact that comparing different processes (by changing the spin or isospin variable) in a single kinematical and geometrical situation may to a large extent eliminate the uncertainties related to the distortions [18]. Hence, using this kind of comparison one may check whether and to what extent the medium modifications of nucleon and mesons properties are reflected in the spin observables of quasifree scattering. One such case is given by coplanar quasifree scattering with polarized incident protons in a single kinematical and geometrical situation by varying the polarization of the incident proton.

The effect of medium modification of the nucleon and mesons masses on the differential cross sections and on spin observables of proton-nucleus elastic scattering has been recently investigated in Ref. [6] by using the Brown and Rho hadronic scaling law [7]. The modification of the meson masses removes the nuclear radius discrepancy 
which persistently occurred in the analysis of the nonrelativistic impulse approximation (NRIA) when empirical nuclear densities obtained from electron scattering are employed. Moreover, the modified meson masses do not spoil the success achieved with the relativistic impulse approximation (RIA) of Ref. [19] on spin observables.

The relative success in accessing medium modifications by means of elastic [20] and quasielastic [21,22] proton scattering motivated us to consider quasifree $(\vec{p}, 2 p)$ scattering to investigate the medium effects on the spin observables $[23,24]$. Compared with elastic nuclear scattering, the quasifree processes are very simple; while the first one deals with the superposition of scattering amplitudes of all nucleons of the nucleus, the last one deals basically with the scattering amplitude of a single nucleon in the nucleus.

Medium effects have been introduced [25] in the treatment of quasifree processes using the density-dependent $t$-matrix interaction of von Geramb and Nakano. It was found that they increase the cross sections somewhat, but scarcely change the analyzing powers. In the present paper we are essentially concerned with the analyzing powers since there seem to exist discrepancies between the experimental results and theoretical predictions [26]. For our analysis of the influence of the medium on the interaction between the incident and knocked-out nucleon, we shall select certain relations between experimental results in which the effects of the distortions are likely to cancel out.

A recent new development in the treatment of $(\vec{p}, 2 p)$ reaction is the use of relativistic distorted impulse approximations (RDIA) [27]. The relativistic calculations include elastic distortions described by relativistic optical potentials with complex vector and scalar potentials, and Dirac-Hartree-like mean field potentials for the nuclear structure. More recently [28], recoil effects have been incorporated in the RDIA calculation. The general result of the relativistic calculations is that they clearly improve the theoretical description of several aspects of the reactions. However, there remain discrepancies mainly related to spin observables at some geometries. In this sense, our study is complementary to the relativistic calculations and might indicate the importance of medium modifications of the basic nucleon properties to be included in a complete calculation.

During the discussion of this paper the following (perhaps somewhat disillusioning) point should be kept in mind. The total state of the knockout process has at least $3(A+1)$ quark degrees of freedom. It is clear that a truly microscopic theory of such a system is impossible. In the impulse approximation the number of participating particles is reduced to three: the incoming and knocked-out nucleon and the residual nucleus. It is most remarkable that with this extreme neglect of degrees of freedom several important characteristics of the knockout reaction, as energy and angular correlations, still can be semi-quantitatively described. This is the main virtue of the various types of impulse approximations.

On the other hand, it is obvious that, if there is a serious experimental deviation from a prediction of an impulse approximation, it might often not be clear which ones of the overwhelming number of neglected degrees of freedom should be invoked to supplement the used version of the impulse approximation. (See, for example, Refs. [28-31].)

In the following section we briefly review the usual formalism for treating quasifree $(\vec{p}, 2 p)$ scattering and compare the experimental data with the theoretical predictions. In Sec. III we use the one-boson exchange Bonn $[32,33]$ potential model to investigate the roles which the different mesons play for the spin observables relevant to the quasifree cross section. The effects of modifications of the masses of the nucleons and mesons and of the mesonnucleon coupling constants on the spin observables are investigated in Sec. IV. There we also study the implications of these modifications for the interpretation of the available experimental data. Our conclusions and future perspectives are presented in Sec. V.

\section{QUASIFREE ( $\vec{p}, 2 p)$ SCATTERING}

In this section we briefty summarize the formalism generally used to calculate the quasifree correlation cross section $[14,15]$ to make the present paper self-contained and to clarify our later arguments. Therefore, we focus our attention just on those aspects relevant to these purposes. We also show that in some special cases the $p p$ analyzing power in medium is directly given by the asymmetries of the $(\vec{p}, 2 p)$ reactions. At the end of this section we discuss the experimental data used to detect nuclear medium modifications of nucleon and meson properties.

The correlation cross section for quasifree scattering in the factorized distorted wave impulse approximation (DWIA) is given by

$$
\frac{d^{5} \sigma}{d \Omega_{1} d \Omega_{2} d E}=K F \frac{d \sigma_{p p}}{d \Omega}\left(E_{0}, \theta, P_{\mathrm{eff}}\right) G\left(\vec{k}_{3}\right) .
$$

Here $K F$ is a kinematical factor. The indices 0,1 , and 2 refer to the incoming and the two emerging particles, respectively, and 3 to the nuclear (ejected) proton. The nucleon-nucleon cross section, $d \sigma_{p p} / d \Omega\left(E_{0}, \theta, P_{\text {eff }}\right)$, is taken at energy $E_{0}$ and angle $\theta$ defined in the centerof-mass system corresponding to the quasifree collision. $G\left(\vec{k}_{3}\right)$ is the distorted momentum distribution of the nuclear proton, with $\vec{k}_{3}=\vec{k}_{1}+\vec{k}_{2}-\vec{k}_{0}$ (equating the negative recoil momentum of the residual nucleus) by momentum conservation.

In the impulse approximation, one assumes that the nuclear medium does not affect the violent nucleonnucleon knockout process. In this case, $d \sigma_{p p} / d \Omega$ is the center-of-mass free cross section for nucleons 0,1 , and 2 with their actual momenta and polarizations in the laboratory system, while the ejected nucleon, 3, has an effective polarization inside the nucleus, represented by $P_{\text {eff }}$.

A free $p p$ cross section has been used to calculate the quasifree cross sections along the years $[14,15,18]$. In this paper we perform an exploratory study about the consequences of relaxing the impulse approximation by using a medium modified $p p$ cross section. An attempt in this 
direction, made by Kudo and Miyazaki [25] by introducing medium effects using a density-dependent $t$ matrix, has scarcely changed the analyzing power.

In the derivation of the cross section [Eq. (1)] besides the impulse approximation for the scattering matrix element of the knockout process, the factorization assumption has been used. That is, fixed average values for the nucleon-nucleon matrix elements have been taken, in spite of the fact that, because of the distortion, the momentum and energy values of the nucleon-nucleon collision in the nucleus have a certain spread around the asymptotic ones. For nucleon-nucleon quasifree scattering at a few hundred $\mathrm{MeV}$ the factorization approximation has been shown to be a good approximation, as long as one avoids those parts of the momentum distributions which are mainly made up of multiple scattered nucleons. These are the regions where the undistorted momentum distributions vanish or are very small [18]. This is an important restriction which shall come up again when we analyze the available experimental data.

These and other assumptions and approximations used to deduce the factorized cross section given by Eq. (1) are extensively discussed in the literature. They include the distortions of the incoming and outgoing nucleons, the off-shell effects, and short range correlations. From the detailed studies over the years, the picture that comes out is that the most doubtful approximation refers to the strong distortions for the incoming and outgoing nucleons. These have been treated via optical potentials, with or without the spin-orbit term. The distortion may reduce the quasifree cross section by one order of magnitude! In contrast, in most cases the spin dependence of the distortion is not too strong [34] and the off-shell effects are relatively small [14-18]. We shall come back to this point.

To avoid uncertainties caused mainly by the distortion, it is desirable to work with ratios of quasifree processes with similar geometrical and kinematical conditions. That is the case for different measurements in a single kinematical and geometrical situation by varying the polarization of the incident beam or the isospin of the ejected nucleon [18]. If the incident polarization is changed, a suitable experimental quantity is the asymmetry defined by

$$
A=\frac{d \sigma^{(+)}-d \sigma^{(-)}}{d \sigma^{(+)}+d \sigma^{(-)}}
$$

where the + and - signs indicate the spin direction of the incoming proton. Using the factorized DWIA, the asymmetry is given entirely in terms of the ratio of protonproton cross sections, with polarizations $P_{0}$ and $P_{\text {eff }}$ orthogonal to the scattering plane ${ }^{2}[35]$ :

$$
\begin{aligned}
\frac{d \sigma}{d \Omega}\left(\theta, T_{\text {rel }}\right)= & I_{0}\left(\theta, T_{\text {rel }}\right)\left[1+\left(P_{0}+P_{\text {eff }}\right) P\left(\theta, T_{\text {rel }}\right)\right. \\
& \left.+P_{0} P_{\text {eff }} C_{n n}\left(\theta, T_{\text {rel }}\right)\right]
\end{aligned}
$$

where $I_{0}\left(\theta, T_{\text {rel }}\right)$ is the free unpolarized $p p$ cross section, and $P\left(\theta, T_{\text {rel }}\right)$ and $C_{n n}\left(\theta, T_{\text {rel }}\right)$ are spin observables for free polarized $p p$ scattering taken at the center-of-mass angle $\theta$ and at the relative kinetic energy $T_{\text {rel }}$. The effective polarization $\left(P_{\text {eff }}\right)$ of the ejected nucleon, caused by the combined influence of the nuclear spin-orbit coupling and the distortion by multiple scatterings, can be quite large in certain geometrical situations. In such a case the matrix element of the corresponding free scattering is, in general, heavily dependent on the polarization of the incoming proton. In this sense the distortion is a desirable mechanism.

The observables $P\left(\theta, T_{\text {rel }}\right)$ and $C_{n n}\left(\theta, T_{\text {rel }}\right)$ are given in terms of the matrix elements of the Wolfenstein matrix as follows [36]:

$$
\begin{aligned}
P\left(\theta, T_{\text {rel }}\right)= & \frac{1}{I_{0}\left(\theta, T_{\text {rel }}\right)} \operatorname{Re}\left[a^{*} e\right] \\
C_{n n}\left(\theta, T_{\text {rel }}\right)= & \frac{1}{2 I_{0}\left(\theta, T_{\text {rel }}\right)}\left\{|a|^{2}-|b|^{2}-|c|^{2}\right. \\
& \left.+|d|^{2}+|e|^{2}\right\} .
\end{aligned}
$$

Another spin observable that we consider in Sec. IV is the depolarization tensor, $D_{n n}\left(\theta, T_{\text {rel }}\right)$, which is given by

$$
\begin{aligned}
D_{n n}\left(\theta, T_{\text {rel }}\right)= & \frac{1}{I_{0}\left(\theta, T_{\text {rel }}\right)}\left\{|a|^{2}+|b|^{2}-|c|^{2}\right. \\
& \left.-|d|^{2}+|e|^{2}\right\} .
\end{aligned}
$$

Substituting Eq. (3) in Eq. (2), we obtain for the asymmetry the following expression:

$$
A=\frac{P\left(\theta, T_{\mathrm{rel}}\right)+P_{\mathrm{eff}} C_{n n}\left(\theta, T_{\mathrm{rel}}\right)}{1+P_{\mathrm{eff}} P\left(\theta, T_{\mathrm{rel}}\right)} P_{0} .
$$

Hence, the effective polarization of the nuclear particle involved in the quasifree scattering can be calculated to a good approximation from the experimental asymmetry $\left(A_{\exp }\right)$ by inverting Eq. (7):

$$
P_{\text {eff }}=\frac{A_{\text {exp }}-P_{0} P\left(\theta, T_{\mathrm{rel}}\right)}{P_{0} C_{n n}\left(\theta, T_{\mathrm{rel}}\right)-A_{\mathrm{exp}} P\left(\theta, T_{\mathrm{rel}}\right)} .
$$

There is a simple prediction one can make for the case of good shell model nuclei, such as ${ }^{16} \mathrm{O}$ and ${ }^{40} \mathrm{Ca}$ : namely, the effective polarizations of the nucleons in two subshells split by the spin-orbit interaction should vanish, to a good approximation, that is [37]

$$
(l+1) P_{\mathrm{eff}}^{j=l+1 / 2}+l P_{\mathrm{eff}}^{j=l-1 / 2} \sim 0 .
$$

This relation agrees with actual distorted wave calculations and is nearly independent of the optical and shell model potentials which generate the distortions and single-particle wave functions, because they are nearly the same for the sub-shells.

Up to now we have just reviewed the usual theoretical treatment of quasifree scattering. An interesting aspect not sufficiently explored in the literature $[15,26,38]$ is to consider special cases for which the effective polarization

\footnotetext{
${ }^{2}$ We consider coplanar quasifree scattering.
} 
of the ejected nucleon is zero. In these cases Eq. (7) leads to $^{3}$

$$
P\left(\theta, T_{\text {rel }}\right)=A_{\text {exp }} .
$$

This means that it is possible to extract the $p p$ analyzing power $\left(P\left(\theta, T_{\mathrm{rel}}\right)\right)$ in medium from the asymmetries of quasifree $(\vec{p}, 2 p)$ reactions. This represents probably the most direct way to get information of the $p p$ analyzing power in medium.

One possibility is to consider the knockout of $s$-state protons. The effective polarization of an $s$-state nucleon is zero since there is no spin-orbit coupling. However, as the momentum distributions for $s$ states peak at a momentum smaller than for others states, the knockout takes place in less dense regions and we do not expect a large medium effect in these states. Another problem is that when working on the steep slope of the $s$-state momentum distribution curve it is not safe to neglect the spin-orbit distortion.

Let us consider then other states and look for special kinematical and geometrical conditions such that $P_{\text {eff }}=0$. For a fixed geometry and kinematics, the values of $\theta$ and $T_{\text {rel }}$ necessary to calculate the asymmetries of the $l+1 / 2$ and $l-1 / 2$ states are not exactly the same, due to the different binding energies of these states. However, since this difference is small and $C_{n n}\left(\theta, T_{\mathrm{rel}}\right)$ and $P\left(\theta, T_{\mathrm{rel}}\right)$ are smooth functions of energy and angle, one has that $C_{n n}^{l+1 / 2} \approx C_{n n}^{l-1 / 2}$ and $P^{l+1 / 2} \approx P^{l-1 / 2}$ to a good approximation. [Here $C_{n n}^{l+1 / 2}$ means the value of $C_{n n}\left(\theta, T_{\text {rel }}\right)$ which enters in Eq. (7) to calculate the asymmetry of the $(l+1 / 2)$ state, and so on.] Within this approximation, $A_{\exp }^{l+1 / 2}=A_{\exp }^{l-1 / 2} \mathrm{im}-$ plies $P_{\mathrm{eff}}^{l+1 / 2}=P_{\mathrm{eff}}^{l-1 / 2}$, in contradiction with Eq. (9), except when $P_{\mathrm{eff}}^{l+1 / 2}=P_{\mathrm{eff}}^{l-1 / 2}=0$. Hence, for those kinematical and geometrical conditions for which the asymmetries of quasifree scattering in two sub-shells split by the spin-orbit interaction are equal $\left(A_{\exp }^{l+1 / 2}=A_{\exp }^{l-1 / 2}\right)$, the effective polarization of the nucleons involved in the quasifree collision should be to a good approximation equal to zero $\left(P_{\text {eff }}^{l+1 / 2} \sim P_{\text {eff }}^{l-1 / 2} \sim 0\right)$. One may therefore extract from Eq. (10) the $p p$ analyzing power in medium from the experimental $(\vec{p}, 2 p)$ asymmetries, by looking for those points where the curve for $A_{\exp }^{l+1 / 2}$ crosses the curve for $A_{\exp }^{l-1 / 2}$. At these special points, $A_{\text {exp }}^{l+1 / 2}=A_{\text {exp }}^{l-1 / 2}=\tilde{P}\left(\theta, T_{\text {rel }}\right)$, where $\tilde{P}\left(\theta, T_{\text {rel }}\right)$ is the $p p$ analyzing power in medium.

Kitching et al. [39] have performed an extensive series of measurements of the asymmetry for the ${ }^{16} \mathrm{O}(\vec{p}, 2 p)^{15} \mathrm{~N}$ reaction in a coplanar geometry with $200 \mathrm{MeV}$ incoming protons with polarizations orthogonal to the scattering plane (normalized to $100 \%$ ). Some of the TRIUMF experimental asymmetries [39] for $200 \mathrm{MeV}$ coplanar $(\vec{p}, 2 p)$ scattering on ${ }^{16} \mathrm{O}$, resulting in the $j=1 / 2$ ground state and the $j=3 / 2$ first excited state of ${ }^{15} \mathrm{~N}$, are shown in

\footnotetext{
${ }^{3}$ For polarized incident beam normalized to $100 \%\left(P_{0}=1\right)$.
}

Fig. 1. ${ }^{4}$ The reader may see in Fig. 1 that there is an appreciable reduction of the analyzing power in medium looking for the special cases where $A_{\exp }^{1 / 2}=A_{\exp }^{3 / 2}$. At these points the asymmetries yield the analyzing power in medium, according to Eq. (10). On the other hand, the free $P\left(\theta, T_{\text {rel }}\right)$ values are indicated by the dashed curves in this same figure and it is clear that the in medium value is smaller than the free one for the nonsymmetrical geometries $\left(\theta_{1} \neq \theta_{2}\right)$. For $\theta_{1}=\theta_{2}$ the free $P\left(\theta, T_{\text {rel }}\right)$ values are small regardless, and not too much can be said.

The effective polarizations calculated [26] from these experimental asymmetries using Eq. (8) with $P$ and $C_{n n}$ for free scattering are reproduced in Fig. 2. In this figure the effective polarization of the $3 / 2$ state is already multiplied by -2 to check the theoretical prediction of Eq. (9): $P_{\text {eff }}^{1 / 2}=-2 P_{\text {eff }}^{3 / 2}$. Agreement between theory and experiment means that the two curves should be on top of each other. For the cases $\theta_{1}=\theta_{2}$ the agreement is excellent. For $\theta_{1} \neq \theta_{2}$ there are large discrepancies. As was remarked, for symmetrical angles, for reasons of symmetry, $P\left(\theta, T_{\text {rel }}\right)$ is small. For asymmetrical angles, $P\left(\theta, T_{\text {rel }}\right)$ is typically 0.3 and the the fits are poor. (See the dashed curves in Fig. 1.)

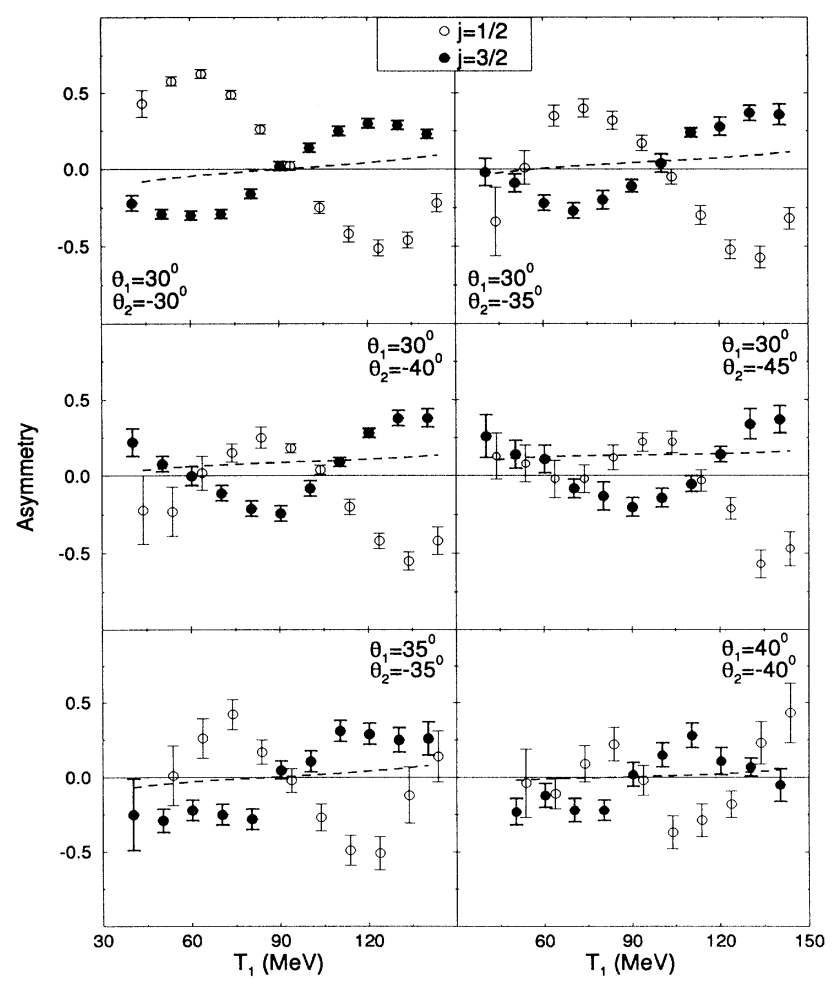

FIG. 1. Experimental asymmetries [39] for $200 \mathrm{MeV}$ coplanar $(\vec{p}, 2 p)$ scattering on the $p$ states of ${ }^{16} \mathrm{O}$. The dashed curves correspond to the free $P\left(\theta, T_{\text {rel }}\right)$ values.

\footnotetext{
${ }^{4}$ We select cases for which most of the experimental data are not at the momentum distributions minima, to avoid uncertainties coming from the multiple scatterings [26].
} 


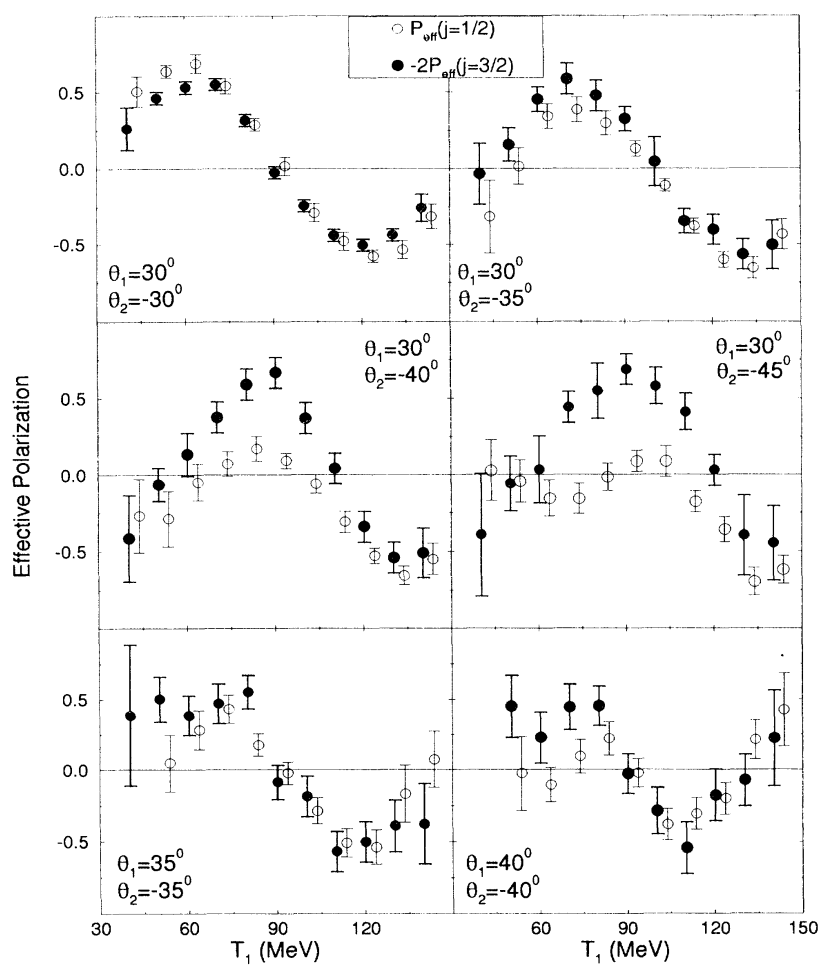

FIG. 2. The effective polarization calculated [26] from the TRIUMF measurements shown in Fig. 1. The effective polarizations of the $j=3 / 2$ state are multiplied by -2 to check Eq. (9).

In Ref. [26] an empirical observation was made: if one sets arbitrarily $P\left(\theta, T_{\mathrm{rel}}\right)=0$ and does not change the value of $C_{n n}\left(\theta, T_{\text {rel }}\right)$, Eq. (8) describes quite well the experimental data for both the asymmetrical and symmetrical cases. In fact, assuming $P\left(\theta, T_{\text {rel }}\right) \approx 0$ in Eq. (7), for nonvanishing effective polarization, one has $\left(P_{0}=1\right)$

$$
\frac{A^{l+1 / 2}}{A^{l-1 / 2}}=\frac{P_{\mathrm{eff}}^{l+1 / 2} C_{n n}^{l+1 / 2}\left(\theta, T_{\mathrm{rel}}\right)}{P_{\mathrm{eff}}^{l-1 / 2} C_{n n}^{l-1 / 2}\left(\theta, T_{\mathrm{rel}}\right)} .
$$

This means that the agreement between theory and experiment achieved in Ref. [26] remains true even if $C_{n n}^{l \pm 1 / 2}\left(\theta, T_{\mathrm{rel}}\right)$ is modified in medium as long as the $C_{n n}$ 's ratio for $j=l+1 / 2$ and $j=l-1 / 2$ remains approximately equal to unity.

The situation described above is not restricted to the ${ }^{16} \mathrm{O}$ nucleus. The measured asymmetries [40] for the reaction ${ }^{40} \mathrm{Ca}(p, 2 p){ }^{39} \mathrm{~K}$ at $200 \mathrm{MeV}$ indicate also a reduction of $P\left(\theta, T_{\text {rel }}\right)$ in medium for a nonsymmetrical geometry, as can be seen in Fig. 3. Again the values for $A_{\text {exp }}^{1 / 2}=A_{\text {exp }}^{3 / 2}$, which give $P\left(\theta, T_{\text {rel }}\right)$ in medium, are much smaller than the free $P\left(\theta, T_{\text {rel }}\right)$ values. Moreover, the effective polarization extracted from these asymmetries using Eq. (8) show a similar behavior as for ${ }^{16} \mathrm{O}$, that is, for symmetrical angles [small values for $P\left(\theta, T_{\text {rel }}\right)$ ] Eq.

(8) describes well the results while for the asymmetrical ones the agreement is poor, as can be seen in Fig. 4 .

The asymmetries have also been measured for ${ }^{40} \mathrm{Ca}(\vec{p}, 2 p)$ populating the $2 s_{1 / 2}$ hole state in ${ }^{39} \mathrm{~K}$. In

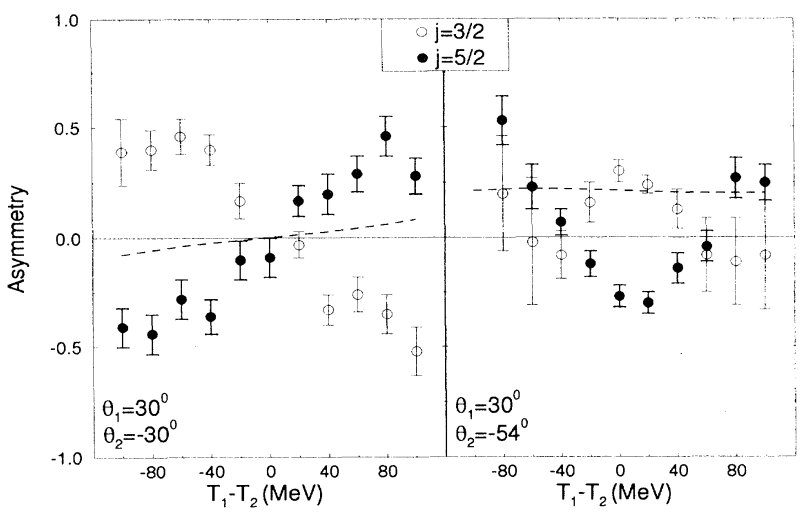

FIG. 3. Experimental asymmetries [40] for $200 \mathrm{MeV}$ coplanar $(\vec{p}, 2 p)$ scattering on the $d$ states of ${ }^{40} \mathrm{Ca}$. The dashed curves correspond to the free $P\left(\theta, T_{\text {rel }}\right)$ values.

this case there is a much smaller reduction (if any) of $P\left(\theta, T_{\text {rel }}\right)$ in medium. However, as has been mentioned, the knockout of $2 s$ states occurs in less dense regions of the nucleus and the effect of the nuclear medium is not expected to be large [38].

The analyzing powers and cross sections for these reactions have been calculated [27] within the framework of the DWIA, including both the effect of the spin-orbit interaction for the distorted waves and off-shell effects in the proton-proton scattering using antisymmetrized $t$ matrix elements calculated with an effective relativistic Love-Franey nucleon-nucleon interaction. The results of the calculations for the $p$-state knockout in ${ }^{16} \mathrm{O}$ agree semiquantitatively with the data. However, it appears that for the ${ }^{16} \mathrm{O}(\vec{p}, 2 p)$ reaction the nonsymmetrical geometry considered $\left(20^{\circ}-65^{\circ}\right)$ shows an agreement of less quality than the two symmetrical ones $\left(30^{\circ}-30^{\circ}\right.$ and $40^{\circ}-$ $\left.40^{\circ}\right)$. For the $s$-state knockout there is a significant difference that has not yet been explained. It would be interesting to know the results which one would get with this treatment for the cases showing discrepancies in our

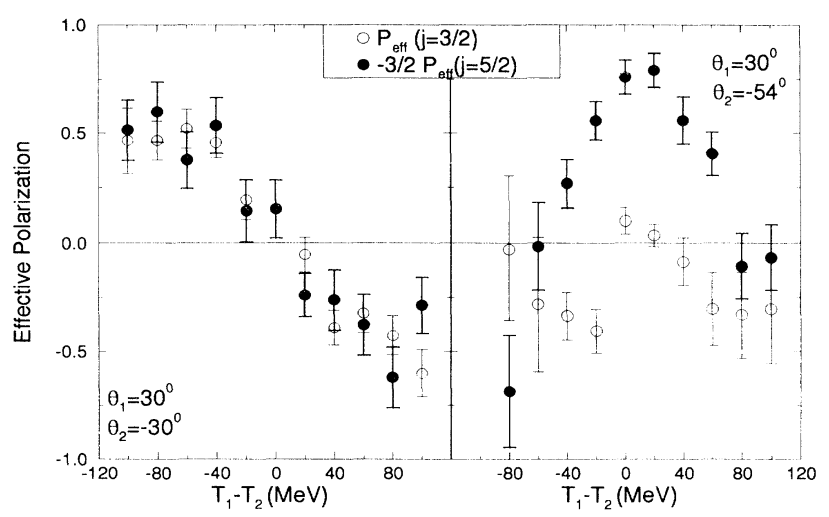

FIG. 4. The effective polarization calculated from the TRIUMF measurements shown in Fig. 3. The effective polarizations of the $j=5 / 2$ state are multiplied by $-3 / 2$ to check Eq. (9). 
analysis $\left(30^{\circ}-40^{\circ}\right.$ and $30^{\circ}-45^{\circ}$ for ${ }^{16} \mathrm{O}$, and $30^{\circ}-54^{\circ}$ for ${ }^{40} \mathrm{Ca}$ at $200 \mathrm{MeV}$ ), as well as for the $2 s$ state in ${ }^{40} \mathrm{Ca}$.

The experimental evidence of a reduction of $P\left(\theta, T_{\text {rel }}\right)$ and the partial nonvalidity of Eq. (9) in medium sets strong constraints on medium modifications of the nucleon-nucleon interaction, as we shall now discuss. In the next section we use the Bonn one-boson exchange model of the nucleon-nucleon interaction to relate the spin observables relevant to quasifree scattering to the properties of the exchanged mesons.

\section{THE NUCLEON-NUCLEON INTERACTION, MESON PROPERTIES, AND SPIN OBSERVABLES}

The free $N N$ interaction is well described by potentials derived from meson exchange models. In this paper we use one of the most successful meson-exchange models, namely the Bonn potential [33]. For the present purposes, it is sufficient to use the one-boson exchange potential (OBEP) which includes $\sigma, \vec{\delta}, \eta, \vec{\pi}, \omega$, and $\vec{\rho}$ meson exchanges.

In order to get some understanding of the contribution of each exchanged meson to the spin observables, we do the following. We use the parameters of the Bonn potential which fit the experimental phase shifts (table 5 of Ref. [32]) and calculate the observables $P$ and $C_{n n}$. Then, we recalculate these spin observables setting the coupling constant of a given meson equal to zero, without changing any other parameter. In this way, it is possible to evaluate the importance of any particular meson to $P$ and $C_{n n}$. The results are shown in Fig. 5. The first fact one learns from this figure is that, not surprisingly, the most important contributions to these observables come basically from two mesons: the $\sigma$ and the $\omega$. (The $\pi$ meson contributes to the observables at low energies only; mainly to $C_{n n}$.) The other important conclusion is that the $\sigma$ meson is the crucial one for $P$. Although the absence of the $\omega$ meson makes the absolute value of $P$ smaller than its experimental value, the absence of the $\sigma$ changes the sign of $P$ with respect to its true value. The observable $C_{n n}$ is sensitive to both $\sigma$ and $\omega$ mesons; the $\pi$ meson is relevant at relatively low energies only.

The crucial observation that the $\sigma$ meson is the most important meson for the observable $P$ may lead us to understand the reduction of $P$ in medium discussed in Sec. II. The potential generated by this meson has central and spin-orbit components. Since a central potential cannot produce a polarization, it appears that the spinorbit component of the nucleon-nucleon potential should be much weaker in the nucleus than in free space.

It seems then that a reduction of $P$ in medium may be associated to the change of the properties of the $\sigma$ meson in the nucleus. It is interesting to note that in a recent relativistic density-dependent Hartree approach for finite nuclei, where the coupling constants of the relativistic Hartree-Lagrangian are made density dependent [11], it was found that $g_{\sigma N N}$ and $g_{\omega N N}$ are of the order of $40 \%$ smaller in medium than in free space.

In order to investigate medium effects on $P$ and $C_{n n}$, we use the Bonn potential [33] to generate $p p$ phase shifts to be used in the calculation of $P$ and $C_{n n}$. However the input parameters (masses and/or coupling constants) are changed according to some prescription.

Although much effort [1-12] has been devoted to the question of medium modifications of the hadronic properties, not too much has been concluded yet. There is a scaling conjecture for hadron properties at finite densities suggested by Brown and Rho [7] based on arguments of partial restoration of chiral symmetry in nuclei. According to this, hadron masses scale as

$$
\begin{aligned}
& \frac{m_{N}^{*}}{m_{N}} \approx \frac{m_{\sigma}^{*}}{m_{\sigma}} \approx \frac{m_{\rho}^{*}}{m_{\rho}} \approx \frac{m_{\omega}^{*}}{m_{\omega}} \approx \frac{f_{\pi}^{*}}{f_{\pi}} \equiv \xi, \\
& \frac{m_{\pi}^{*}}{m_{\pi}} \approx 1,
\end{aligned}
$$

where $f_{\pi}$ is the pion decay constant, $m_{N}, m_{\rho}, m_{\omega}$, and $m_{\pi}$ are the masses of the nucleon, $\vec{\rho}, \omega$, and $\vec{\pi}$ mesons, respectively, and $m_{\sigma}$ is the mass of the effective scalar $\sigma$ meson. The asterisk denotes the value of these quantities in nuclear medium.

Other authors have also discussed hadronic scaling law for the masses based on QCD arguments $[2,4,9,10,12]$. Kusaka and Weise [9] have concluded that the Brown and Rho scaling law is not realized for reasonable parameter changes. However, Gao et al. [12], based on the

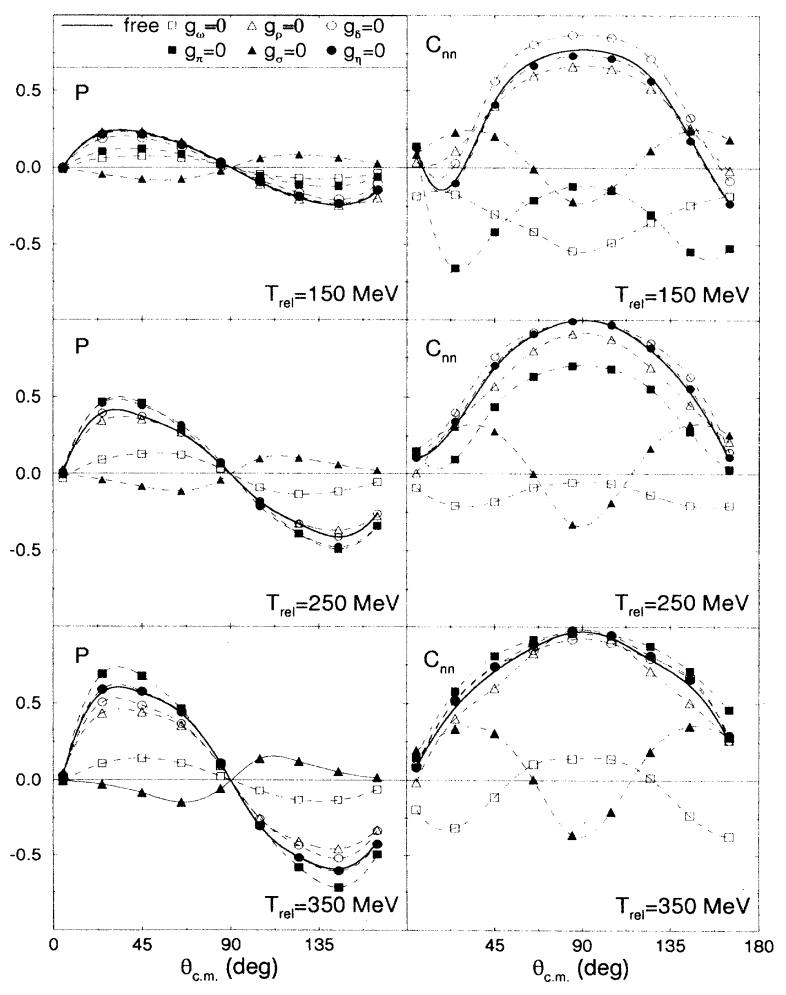

FIG. 5. The observables $P\left(\theta, T_{\text {rel }}\right)$ and $C_{n n}\left(\theta, T_{\text {rel }}\right)$ calculated with the Bonn potential with parameters that fit the experimental phase shifts for free scattering on protons (solid curves) and turning off different mesons. 
thermofield dynamical theory, have concluded that for $\rho<4 \rho_{0}$, where $\rho_{0}$ is the saturation density, the Brown and Rho conjecture should be correct. Hatsuda and Lee [10] have obtained a linear decrease of the masses as a function of density; their results seem to support the Brown and Rho scaling law. Although the validity of the Brown and Rho law is still controversial, we take it as a starting point to investigate the behavior of the observables with changed hadronic mass.

Another open question is the value assumed by $\xi$ in Eq. (12). We have taken it in the range 0.6-0.9.

With respect to the variations of coupling constants, the situation is even more controversial $[8,11]$. As has been mentioned, it was found by Brockmann and Toki [11] in a relativistic density-dependent Hartree approach that the $g_{\sigma N N}$ and $g_{\omega N N}$ are $\sim 40 \%$ smaller in medium than in free space. Banerjee's toy model, based on a chiral confining model, leads to a reduction of $g_{\sigma N N}$ with density, while $g_{\omega N N}$ and $g_{\rho N N}$ increase at some low rate with the density. There is still a scaling law derived by Banerjee [8], using the results of McGovern, Birse, and Spanos [5], which leads to an increase of $g_{\sigma N N}$ and $g_{\omega N N}$ in medium. As we do not have a definitive prescription for changing the coupling constants in medium, we assume that $g_{\sigma N N}$ and $g_{\omega N N}$ decrease in medium [11] according to

$$
\frac{g_{\sigma N N}^{*}}{g_{\sigma N N}}=\frac{g_{\omega N N}^{*}}{g_{\omega N N}}=\chi
$$

where $\chi$ is assumed in the range $0.6<\chi<0.9$.

We also consider simultaneous variations of masses and coupling constants by taking Eqs. (12) and (13) simultaneously.

In summary, we consider three prescriptions:

(i) only the masses are changed according to Eq. (12).

(ii) only the $\sigma N N$ and $\omega N N$ coupling constants are changed according to Eq. (13).

(iii) the $\sigma N N$ and $\omega N N$ coupling constants and masses are changed simultaneously according to Eqs. (12) and (13).

We have not considered medium modifications of masses and/or coupling constants of the mesons $\vec{\rho}, \eta$, and $\vec{\delta}$ since their contributions to $P$ and $C_{n n}$ at the energies we are considering are much smaller than the ones from $\sigma$ and $\omega$, as can be seen in Fig. 5. With respect to the pion, since it is a Goldstone boson, its mass presumably changes only slowly with density $[3,6]$ and modifications on the $g_{\pi N N}$ affect the spin observables only at low energies (Fig. 5). We have checked our results against variations of the pion mass and coupling constant. These modifications do not change our conclusions.

In Fig. 6 we show the effect on the observables $P$ and $C_{n n}$ of changing the masses and/or coupling constants according to the three prescriptions above, taking $\xi=$ 0.7 and $\chi=0.75$. The figures show that in all three prescriptions there is a reduction of $P\left(\theta, T_{\text {rel }}\right)$ in medium compared to the free value. For other values of $\xi$ and $\chi$

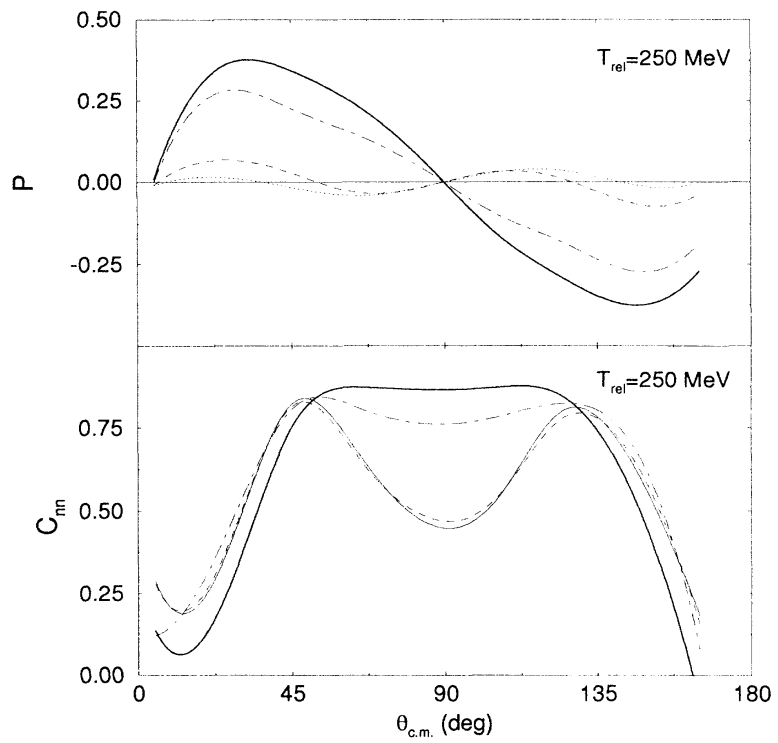

FIG. 6. The observables $P\left(\theta, T_{\text {rel }}\right)$ and $C_{n n}\left(\theta, T_{\text {rel }}\right)$ calculated with the Bonn potential. The solid curves correspond to parameters that fit the experimental phase shifts. The dashed curves correspond to scaling the masses [Eq. (12) with $\xi=0.7]$, dot-dashed curves correspond to scaling coupling constants [Eq. (13) with $\chi=0.75$ ], and dotted curves correspond to scaling masses and coupling constants with $\xi=0.7$ and $\chi=0.75$.

the results are basically the same except that the curves cross the axis in slightly different places. The reduction increases, as $\xi$ and/or $\chi$ decrease. $C_{n n}$ is reduced for $45^{\circ} \lesssim \theta \lesssim 135^{\circ}$ and enhanced for other values of $\theta$ in all three prescriptions.

\section{MEDIUM MODIFICATIONS AND QUASIFREE REACTIONS}

In this section we analyze the implications of the medium modifications for the $(\vec{p}, 2 p)$ asymmetries.

We have calculated the values of $P$ and $C_{n n}$ with the three prescriptions explained in Sec. III, taking $0.6<$ $\xi, \chi<0.9$. The effective polarizations are then calculated by using the experimental asymmetries in Eq. (8). A remarkably good agreement between these effective polarizations and the theoretical prediction, Eq. (9), is obtained when one changes simultaneously masses and coupling constants and takes $\xi=0.7$ and $\chi=0.75$. The results are shown in Figs. 7 and 8 for the ${ }^{16} \mathrm{O}(\vec{p}, 2 p){ }^{15} \mathrm{~N}$ and ${ }^{40} \mathrm{Ca}(\vec{p}, 2 p){ }^{39} \mathrm{~K}$, respectively. These figures represent the same quantities as in Figs. 2 and 4 , but the $P$ and $C_{n n}$ are calculated with the modified nucleon-nucleon interaction. $^{5}$

\footnotetext{
${ }^{5}$ As for Figs. 2 and 4, agreement between theory and experiment means that the two curves should be on top of each other.
} 


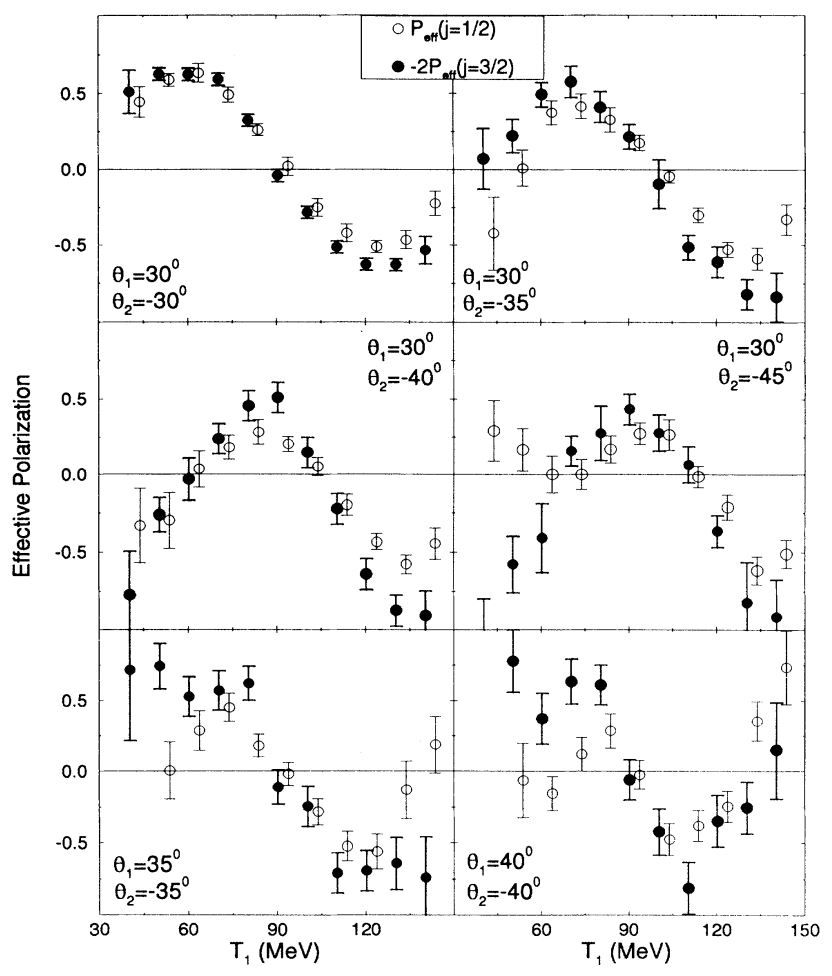

FIG. 7. The same quantities as in Fig. 2, but with masses and coupling constants changed according to Eq. (12) $(\xi=0.7)$ and Eq. $(13)(\chi=0.75)$.

The conclusion one can draw from these figures is that the modifications of nucleon and meson properties clearly affect the spin observables of the reaction in a significant way. As mentioned in the introduction, although relativistic effects including retardation lead to improvement on the calculated $(\vec{p}, 2 p)$ cross sections, there still remain discrepancies for spin observables in some geometrical regions. In this sense, it might be worthwhile to further

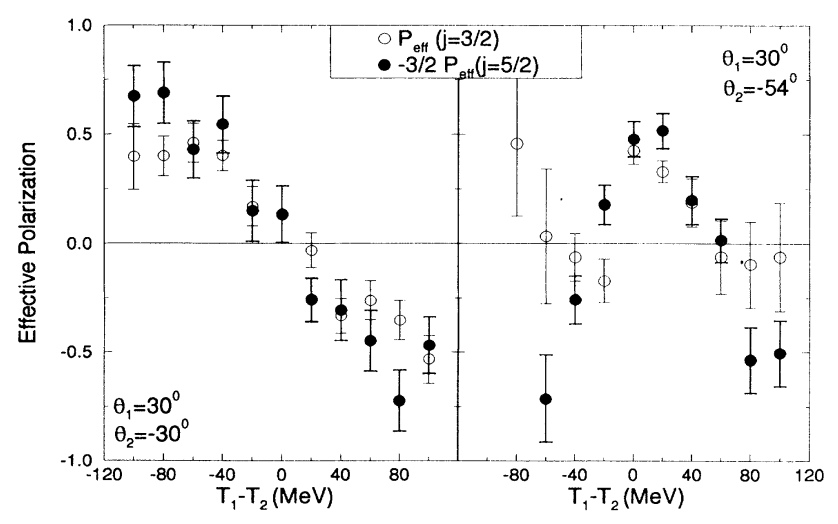

FIG. 8. The same quantities as in Fig. 3 but with masses and coupling constants changed according to Eq. (12) $(\xi=0.7)$ and Eq. $(13)(\chi=0.75)$. investigate the inclusion of medium modifications on the basic interaction process.

As has been mentioned, in Ref. [26] the discrepancies have been eliminated by taking $P$ arbitrarily equal to zero and using the free space value of $C_{n n}$. In our calculation, for consistency, we assumed that both $P$ and $C_{n n}$ are modified in medium. The value of $P$ turned out to be drastically reduced in medium, but it does not go exactly to zero. The value of $C_{n n}$ is also changed in medium, however it is still a smooth function of energy and angle. As a consequence, the ratio $C_{n n}^{j=l+1 / 2} / C_{n n}^{j=l-1 / 2} \approx 1$ and the agreement is achieved rather independently of the free $C_{n n}$ value.

In an earlier attempt [24] the reduction of $P$ in medium was investigated using a formalism developed by Horowitz and Iqbal [21]. In their formalism, the medium modification are evaluated in a relativistic model where the $N N$ interaction is assumed to depend on the enhancement of the lower components of the nucleon Dirac spinor due to strong scalar and vector nuclear potentials. Although this formalism also leads to a reduction of the $p p$ analyzing power in medium, the effect is too small to eliminate the observed discrepancies. The influence of a depolarization of the incident beam as well as off-shell effects have also been investigated a long time ago [41] and do not explain the discrepancies.

Still lacking is a clear explanation of the fact that in the $2 s$ knockout from ${ }^{40} \mathrm{Ca}$ the reduction of the analyzing power is much smaller than in the $1 p$ and $1 d$ states studied here. Based on the argument that the $2 s$-state knockout occurs in less dense regions of the nucleus one would expect to describe the data with our approach using larger values for $\xi$ and/or $\chi$ compared to the values used for $p$ and $d$ states. Our analysis for this case indicates that $\xi$ and $\chi$ must be larger than 0.9 .

Up to now we have discussed the spin observables which enter in the coplanar $(\vec{p}, 2 p)$ quasifree cross sections, namely $P$ and $C_{n n}$. We observed that the three prescriptions for hadronic scaling laws affect these observables. However, we do not expect to be able to discriminate between the three prescriptions through these observables solely, since the effects always go in the same direction, i.e, when a given prescription leads to an enhancement (reduction) of $P$ or $C_{n n}$, the other two prescriptions lead to an enhancement (reduction) too.

However, the proposed measurement of $(\vec{p}, 2 \vec{p})$ quasifree reactions proposed at IUCF and TRIUMF will have indirect access to another spin observable, namely the depolarization tensor, $D_{n n}$. For this observable, in contrast, the effects of the three prescriptions are quite different, as can be seen in Fig. 9. It is clear that such a measurement might provide severe constraints on medium modifications of hadron properties. ${ }^{6}$

\footnotetext{
${ }^{6}$ Kudo and Tsunoda [42] have calculated the depolarization tensors for the $1 d_{5 / 2}, 1 d_{3 / 2}$, and $2 s_{1 / 2}$ hole states in the ${ }^{40} \mathrm{Ca}(\vec{p}, 2 p){ }^{39} \mathrm{~K}$ at $E=200 \mathrm{MeV}$.
} 


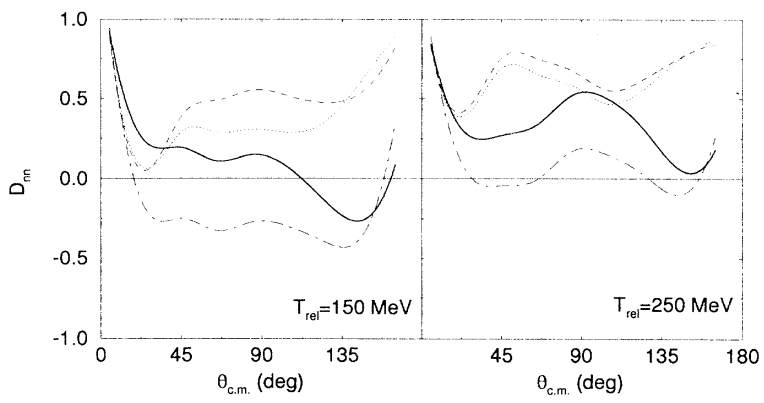

FIG. 9. The observables $D_{n n}$ calculated with the Bonn potential. The convention is the same as in Fig. 6.

\section{CONCLUSIONS}

We have used quasifree knockout reactions to investigate medium modifications of bound nucleons. As remarked in the introduction, care must be taken when the factorized ${ }^{7}$ quasifree $(\vec{p}, 2 p)$ cross sections, as in this paper, are used.

The factorized form of the cross sections has been checked often and it turned out that the best way is to avoid the minima of the momentum distributions of the ejected nucleon (where a large smearing of the momentum happens) and to work with certain ratios of quasifree cross sections to cancel out uncertainties related to the optical potentials. With this care in mind, the factorized cross section shows the advantage of making the physics of the process transparent. For instance, an effective polarization of the ejected nucleon (before the knockout process) is understandable in terms of a combined effect of the spin-orbit interaction and the absorption of the ejected nucleon [13]. As for medium energy, in the angular region needed for the absorption effect, the cross section for protons with parallel spins is much larger than the one for opposite spins, an asymmetry is expected (and detected) for the $(\vec{p}, 2 p)$ process with polarized incident beams.

There is also a theoretical prediction which relates the effective polarizations for nucleons in two subshells split by the spin-orbit interaction. In principle, one could doubt the validity of this prediction since it is based on the factorization approximation. However, it is remarkable that the data agree quite well with this theoretical prediction when the angles of the two emerging particles are equal. When the emerging angles are different some discrepancies show up. These discrepancies have been observed a long time ago [26] and various attempts to explain them have been made on the basis of off-shell effects and depolarization of the incident beam [41], as well as by taking into account the nucleon effective mass inside the nucleus $[23,24]$. To our knowledge, none of these has been successful.

On the basis of a factorized quasifree cross section, we have proposed to extract the $p p$ analyzing power

\footnotetext{
${ }^{7}$ The quasifree cross section is factorized into a product or the momentum distribution of the ejected nucleon times a $p p$ cross section at energy and angles corresponding to the violent interaction.
}

in medium $(P)$ through the asymmetries of $(\vec{p}, 2 p)$ processes. In particular, $P$ is equal to the experimental asymmetries for two subshells split by the spin-orbit interaction for geometrical and kinematical situations such that $A^{j=l+1 / 2}=A^{j=l-1 / 2}$. From the measured asymmetries for $200 \mathrm{MeV}$ coplanar $(\vec{p}, 2 p)$ on $1 p$ states of ${ }^{16} \mathrm{O}$ and $1 d$ states of ${ }^{40} \mathrm{Ca}$, we have observed a reduction of $P$ in medium.

A reduction of the $p p$ analyzing power in medium is also predicted by the Horowitz and Iqbal relativistic treatment [21] of proton nucleus scattering. In this approach a modified $N N$ interaction in medium is assumed due to the effective nucleon mass (smaller than the free mass) which affects the Dirac spinors used in the calculations of the $N N$ scattering matrix. Cross sections and spin observables are modified in medium. For instance, the analyzing power is found to decrease $40 \%$ compared to the free value at $500 \mathrm{MeV}$ for an effective nuclear mass $\sim 15 \%$ smaller than the free value. This treatment is unable to explain the discrepancies under discussion in the quasifree $(\vec{p}, 2 p)$ asymmetries [23,24].

In this paper we have performed an exploratory study towards a possible explanation of the $P$ reduction observed in $(\vec{p}, 2 p)$ scattering in terms of medium modifications of nucleon and meson properties. The first conclusion is that the $\omega$ and especially the $\sigma$ meson give the main contribution for this observable. The next step was to use hadronic scaling laws in our calculations. As this issue is still controversial, in this exploratory study we have considered possible modifications of masses and/or coupling constants for the $\sigma$ and $\omega$ mesons, which are the most important for the spin observables. It turned out that by scaling simultaneously masses and coupling constants we have been able to eliminate the discrepancies observed in the asymmetries of $(\vec{p}, 2 p)$ reactions. We do not know of any other explanation for these discrepancies.

As we have remarked before, it is important to note that our results are obtained within the framework of the DWIA. We have been careful in choosing special experimental results in order to minimize uncertainties related to the DWIA. In recent years, there have been advances in microscopic calculations of optical potentials consistent with multiple scattering theory which go beyond the early first-order calculations [29-31]. A combination with medium modifications of the basic nucleon-nucleon interaction would allow for interesting comparisons with less special experimental data.

Our results indicate that quasifree $(\vec{p}, 2 p)$ reactions might be a powerful tool to investigate medium modifications of bound nucleons and hopefully can be used to discriminate different theoretical predictions. Improvements on the theory and more experimental data at various bombarding energies are clearly needed.

\section{ACKNOWLEDGMENTS}

Support by Conselho Nacional de Desenvolvimento Científico e Tecnológico (CNPq), Fundação de Amparo à Pesquisa do Estado do Rio Grande do Sul (FAPERGS), and Fundação de Amparo à Pesquisa do Estado de São Paulo (FAPESP) is acknowledged. 
[1] For a recent review, see T. Hatsuda, Nucl. Phys. A544, 27c (1992).

[2] V. Bernard, U.G. Meissner, and I. Zahed, Phys. Rev. Lett. 59, 966 (1987).

[3] Y. Nambu, in Festival-Festschrift for Val Teledgdi, edited by K. Winter (Elsevier, New York, 1988).

[4] B.A. Campbel, John Ellis, and K.A. Olive, Phys. Lett. B 235, 325 (1990); Nucl. Phys. B345, 57(1990).

[5] J.A. McGovern, M.C. Birse, and D. Spanos, J. Phys. G 16, 1561 (1990).

[6] G.E. Brown, A. Sethi, and N.M. Hintz, Phys. Rev. C 44, 2653 (1991).

[7] G.E. Brown and M. Rho, Phys. Rev. Lett. 66, 2720 (1991).

[8] M.K. Banerjee, Phys. Rev. 45, 1359 (1992).

[9] K. Kusaka and W. Weise, Phys. Lett. B 288, 6 (1992).

[10] T. Hatsuda and S.H. Lee, Phys. Rev. C 46, R34 (1992).

[11] R. Brockmann and H. Toki, Phys. Rev. Lett. 68, 3408 (1992).

[12] S. Gao, R-K. Su, and P.K.N. Yu, Phys. Rev. C 49, 40 (1994).

[13] Th.A.J. Maris, P. Hillman, and H. Tyrén, Nucl. Phys. 7, 1 (1958); Th.A.J. Maris, Nucl. Phys. 9, 577 (1958/1959).

[14] G. Jacob and Th.A.J. Maris, Rev. Mod. Phys. 38, 121 (1966) and 45, 6 (1973).

[15] P. Kitching, W.J. McDonald, Th.A.J. Maris, and C.A.Z. Vasconcellos, Adv. Nucl. Phys. 15, 43 (1985).

[16] P. Kitching, C.A. Miller, D.A. Hutcheon, A.N. James, W.J. McDonald, J.M. Cameron, W.C. Olsen, and G. Roy, Phys. Rev. Lett. 37, 1600 (1976).

[17] A.N. James, W.J. McDonald, J.M. Cameron, C.A. Miller, D.A. Hutcheon, P. Kitching, G.C. Neilson, G.M. Stinson, and E.D. Earle, Nucl. Phys. A324, 253 (1979).

[18] Th.A.J. Maris, M.R. Teodoro, and E.A.Veit, Phys. Rev. C 20, 446 (1979).

[19] J.A. Tjon and S.J. Wallace, Phys. Rev. C 38, 1085 (1987).

[20] D.P. Murdock and C.J. Horowitz, Phys. Rev. C 35, 1442 (1987).

[21] C.J. Horowitz and J. Iqbal, Phys. Rev. C 33, 2059 (1986).

[22] C.J. Horowitz and D.P. Murdock, Phys. Rev. C 37, 2032 (1988).

[23] B.B. Rodrigues, Master dissertation, Universidade Federal do Rio Grande do Sul, 1992 (unpublished).

[24] G. Krein, B.B. Rodrigues, and E.A. Veit, in Current Top- ics in Nuclear Physics and Quantum Field Theory, edited by D. Dillenburg, G. Jacob, P. Kitching, and C.A.Z. Vasconcelos; Festschrift for Prof. Th.A.J. Maris (Ed. da Universidade, Porto Alegre, 1994), pp. 55-72.

[25] Y. Kudo and K. Miyazaki, Phys. Rev. C 34, 1192 (1986).

[26] Th.A.J. Maris, M.R. Teodoro, and E.A. Veit, Phys. Lett. B94, 6(1980).

[27] E.D. Cooper and O.V. Maxwell, Nucl. Phys. A493, 468 (1989); O.V. Maxwell and E.D. Cooper, ibid. A513, 584 (1990); A574, 819 (1994).

[28] O.V. Maxwell and E.D. Cooper, Nucl. Phys. A565, 740 (1993).

[29] R. Crespo, R.C. Johnson, and J.A. Tostevin, Phys. Rev. C 41, 2257 (1990); 44, R1735 (1991); 46, 279 (1992); 48, 351 (1993).

[30] H.F. Arellano, F.A. Brieva, and W.G. Love, Phys. Rev. Lett. 63, 605 (1989); Phys. Rev. C 41, 2188 (1990).

[31] Ch. Elster, T. Cheon, E.F. Redish, and P.C. Tandy, Phys. Rev. C 41, 814 (1990); C.R. Chinn, Ch. Elster, and R.M. Thaler, ibid. 44, 1569 (1991); 47, 2242 (1993); 48, 2956 (1993).

[32] R. Machleidt, K. Holinde, and Ch. Elster, Phys. Rep. 149, 1(1987).

[33] R. Machleidt, in Computational Nuclear Physics, edited by K. Langanke, J.A. Maruhn, and S.E. Koonin (Springer-Verlag, New York, 1993), Vol. 2, Chap. 1.

[34] C. Schneider, Nucl. Phys. A300, 313 (1978); N.S. Chant, P. Kitching, P.G. Ross, and L. Antonuk, Phys. Lett. 43, 495 (1979).

[35] L.Wolfenstein, Annu. Rev. Nucl. Sci. 6, 43 (1953).

[36] J. Bystrick, F. Lehar, and P. Winternitz, J. Phys. (Paris) 39, 1 (1978).

[37] V.E. Herscovitz, Th.A.J. Maris, and M.R. Teodoro, Phys. Lett. 69B, 33 (1977).

[38] P. Kitching, in [24], pp. 31-44.

[39] P. Kitching, C.A. Miller, W.C. Olsen, D.A. Hutcheon, W.J. McDonald, and A.W. Stetz, Nucl. Phys. A340, 423 (1980).

[40] L. Antonuk, P. Kitching, C.A. Miller, D.A. Hutcheon, W.J. McDonald, G.C. Neilson, and W.C. Olsen, Nucl. Phys. A370, 389 (1981).

[41] E.A. Veit, Ph.D. thesis, Universidade Federal do Rio Grande do Sul, 1981 (unpublished).

[42] Y. Kudo and H. Tsunoda, Prog. Theor. Phys. 89, 89 (1993). 